\title{
Correlación clínica de los hallazgos ultrasonográficos de las uñas y de las articulaciones interfalángicas distales en pacientes con psoriasis
}

Clinical correlation of ultrasound subclinical findings in nails and distal interphalangeal joints in psoriatic patients

\section{Arturo Argote', Humberto Rivera², Freddy Alejandro Suárez ${ }^{3}$, Adriana Gómez ${ }^{4}$, Ximena Wortsman 5}

1. Médico dermatólogo; especialista en Docencia Universitaria. FUCS . Profesor asociado, Fundación Universitaria de Ciencias de la Salud, Hospital de San José, Bogotá, D.C., Colombia.

2. Médico radiólogo. Fundación Santa Fe de Bogotá, Bogotá, D.C., Colombia.

3. Médico dermatólogo, Fundación Universitaria de Ciencias de la Salud, Hospital de San José, Bogotá, D.C., Colombia.

4. Médica dermatóloga, Fundación Universitaria de Ciencias de la Salud, Bogotá, D.C., Colombia.

5. Médica radióloga, especialista en Ultrasonografía Dermatológica, Departamento de Radiología y Departamento de Dermatología, Clínica Servet, Facultad de Medicina, Universidad de Chile, Santiago de Chile, Chile.

\section{RESUMEN}

ОвJETIVo. Describir y correlacionar los hallazgos clínicos y los ultrasonográficos en las uñas y las articulaciones interfalángicas distales, en pacientes con psoriasis y en controles sanos.

DisEÑo. Es un estudio observacional descriptivo. Se incluyeron pacientes mayores de edad, que acudieron a la consulta externa dermatológica del Hospital Universitario de San José. Se conformaron tres grupos de estudio: pacientes con psoriasis cutánea y compromiso ungular, pacientes con psoriasis sin compromiso ungular y pacientes sin enfermedad dermatológica.

Mediante la ecografía, se determinaron el espesor del lecho ungular, el grosor de la placa, el índice de resistencia en las arterias del lecho ungular, los cambios osteotendinosos, las márgenes y las ondulaciones ungulares.

RESUlTADOS. Se analizaron 31 sujetos: 11 con psoriasis y compromiso ungular (55 dedos), 10 casos con psoriasis sin compromiso ungular (50 dedos) y 10 controles sanos (50 dedos). La mediana del espesor del lecho ungular fue mayor entre los grupos con psoriasis (ungular, de 1,9 mm con rango intercuartílico de [RIQ] de 1,6 a 2,2; y cutánea, de 1,9 mm con RIQ de 1,7 a 2,1), comparada con la de los sujetos sanos (de $1,7 \mathrm{~mm}$, con RIQ de 1,5 a 1,9) (p<0,001).

Hubo diferencias significativas en el grosor de la placa ungular entre los tres grupos ( $\mathrm{p}<0,001)$ : con psoriasis ungular, fue de $0,7 \mathrm{~mm}$ con RIQ de 0,7 a 0,9; con psoriasis cutánea sin compromiso ungular, fue de o,8 mm, con RIQ de 0,7 a 0,9; en sujetos sanos fue de $0,6 \mathrm{~mm}$, con RIQ de o,6 a o,7; no se observaron diferencias en los valores del índice de resistencia arterial $(\mathrm{p}=0,12)$.

LimitACiONES. Aunque se incluyeron 155 unidades de análisis, estas corresponden a 31 pacientes. El estudio fue realizado con un equipo de alta resolución que alcanza una frecuencia máxima de $14 \mathrm{MHz}$.
Correspondencia:

Arturo Argote

Email:

arturoargote@hotmail.com

Recibido: 4 de marzo de 2105

Aceptado: 7 de mayo de 2015

Conflictos de interés:

La investigación fue cofinanciada por la Fundación Universitaria de Ciencias de la Salud, convocatoria 2010-4, y MSD Colombia. La toma de ultrasonografías ungulares y HLA B27, fue patrocinada por MSD. La elaboración del protocolo, la fase de recolección de datos, el análisis de resultados y el manuscrito son responsabilidad exclusiva de los autores, sin participación de MSD Colombia. 
CoNCLUSIONES. En pacientes con psoriasis, la ultrasonografía del aparato ungular nos podría dar aportes que contribuirían a la toma de decisiones clínicas tempranas.

PALABRAS CLAVE: ultrasonografía ungular, psoriasis cutánea, psoriasis ungular, ultrasonido, uña.

\section{SUMMARY}

ОвJестіvE: The purpose of this study is to describe the subclinical ultrasound findings in nails and distal interphalangeal joints in psoriatic patients.

METHODS: It was a descriptive observational study. Three study groups were described: patients presenting cutaneous and nail psoriasis (CNP), psoriatic patients without psoriatic nails (CP), and healthy nails (HN).

Ultrasound measurements of the nail bed and nail plate thickness, resistance index in nail bed arteries, osteotendinous changes, ungual plate margins and undulations were performed.

RESULTS: 31 subjects were analyzed: 11 cutaneous and nail psoriasis, 10 cutaneous and 10 healthy nails. The median thickness of the nail bed was higher amongst the groups with psoriasis (CNP 1.9mm IQR [1.6-2.2], CP 1.9mm IQR [1.7-2.1]) compared to HN (1.7mm IQR [1.5-1.9]), ( $\mathrm{p}<0.001)$. The nail plate thickness had significant differences between groups, ( $\mathrm{p}<0.001$ ) (CNP $0.7 \mathrm{~mm}$ IQR [0.7-0.9], CP o.8mm [0.7-0.9], HI o.6mm [0.6-0.7]).

CoNCLUSIONS: In psoriatic patients, ultrasonography of the nail unit provides subclinical information that is not available from the physical examination.

\section{INTRODUCCIÓN}

La psoriasis es una enfermedad inflamatoria asociada a un síndrome metabólico que afecta en promedio al $2 \%$ de la población en los Estados Unidos; solo en ese país, aqueja entre 4,5 y 7 millones de personas ${ }^{1}$. Existen diferencias en la frecuencia frente a otros países. Por ejemplo, en Colombia, en algunos servicios de dermatología, del total de pacientes de consulta externa, el 3 por ciento sufre psoriasis ${ }^{2}$.

La psoriasis ungular es una afección inflamatoria crónica de la matriz y del lecho ungular, que afecta en promedio de 15 a $50 \%$ de los pacientes con psoriasis ${ }^{1}$. Una herramienta útil en la valoración de la psoriasis ungular es el NAPSI (Nail Psoriasis Severety Index) ${ }^{3}$, por medio de la cual se detectan signos clínicos específicos de la matriz y el lecho ungulares, que permiten medir de manera objetiva la intensidad y la extensión de la psoriasis ungular de manos y pies ${ }^{3}$. El diagnóstico clínico preciso requiere dedicación durante el examen físico, más tiempo y valoración dermatoscópica de cada uña ${ }^{4}$. Para el diagnóstico también se utiliza la biopsia de matriz y lecho ungulares, procedimiento asociado a dolor y a posible alteración permanente de de las uñas.
La psoriasis ungular se encuentra muy relacionada con las artritis inflamatorias seronegativas, que pueden comprometer cualquier tipo de articulación, con una mayor predisposición de las articulaciones interfalángicas distales de las manos ${ }^{5,6}$. Existe una importante asociación entre las estructuras músculo-esqueléticas y el aparato ungular; se describe que las entesitis subclínicas son una característica de los pacientes con psoriasis sin artritis psoriática 5 .

El inicio temprano de la enfermedad poliarticular ha sido sugerido como un factor predictor de la enfermedad deformante y erosiva, que causa discapacidad física, funcional y psicosocial ${ }^{5,6}$. En $40 \%$ de los pacientes con artritis psoriática se ha visto una asociación con la presencia de antígenos del complejo mayor de histocompatibilidad: HLA-B13, B17, B38, B39, B27, CW*0602, DR4 y DR77 .

En estudios recientes se han encontrado en la piel de pacientes con psoriasis, abundantes cantidades de IL23, la cual tiene un importante rol en la patogénesis de la psoriasis; a su vez, se encuentra elevada en pacientes con entesitis y con espondiloartropatías ${ }^{2,8,9}$.

El uso actual del ultrasonido de alta resolución como método de cuantificación de la intensidad de la enfermedad ungular y articular, permite obtener imágenes 


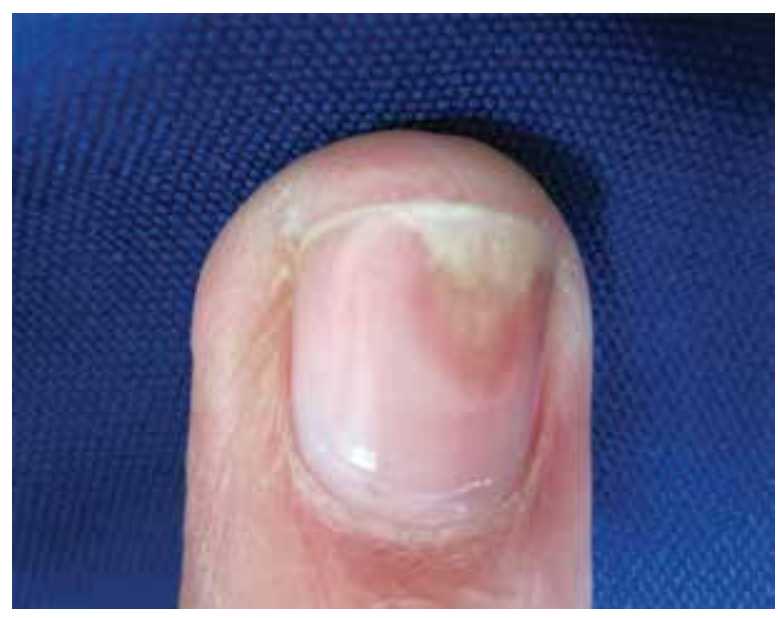

FIGURA 1. Paciente con psoriasis ungular: gota de aceite y onicólisis.

que describen la dinámica y las propiedades tisulares, ayudando en el estudio de muchas afecciones de la piel y las uñas. El ultrasonido de alta resolución es una técnica de imagen en tiempo real, por medio de la cual se obtiene una imagen de buena calidad, con la ventaja de que es un método accesible $\mathrm{e}^{10,11}$.

Los objetivos del presente estudio fueron describir los hallazgos de la ultrasonografía de alta resolución en las uñas y las articulaciones interfalángicas distales, en pacientes con psoriasis ungular y pacientes con psoriasis sin compromiso ungular, haciendo comparaciones con sujetos sanos y correlacionando estos resultados con los hallazgos clínicos, en los grupos descritos.

\section{MÉTODOS}

Se llevó a cabo un estudio observacional descriptivo. Se incluyeron pacientes mayores de 18 años que acudieron a la consulta dermatológica. Se conformaron tres grupos de estudio, con los siguientes criterios de selección.

- Primer grupo: 11 casos con diagnóstico de psoriasis confirmado por biopsia de piel, con evidencia clínica de compromiso ungular y con NAPSI positivo (NAPSI>0), reportado por un dermatólogo.

- Segundo grupo: 10 pacientes con diagnóstico confirmado de psoriasis documentado por biopsia de piel, sin compromiso ungular aparente y NAPSI negativo (NAPSI=0).

- Tercer grupo: 10 sujetos con unidades ungulares sanas según criterio del dermatólogo.
Se excluyeron los pacientes con afección ungular de tipo inflamatorio, infeccioso o traumático, y los que tenían enfermedad coexistente autoinmunitaria, osteodegenerativa o tumoral. Para ello, se tomo biopsia de la placa ungular para descartar otro proceso inflamatorio o infeccioso.

A cada uno de los sujetos que participaron en el estudio se le practicó ultrasonografía de la mano dominante. Para el análisis se obtuvieron datos de 55 dedos en el primer grupo, de 50 dedos en el segundo y de 50 dedos en el tercero.

La valoración clínica de las uñas fue hecha por un dermatólogo con experiencia, utilizando un dermatoscopio (Dermlite $\mathrm{dl} 100^{\circledR}$ ) para calcular el NAPSI, según las categorías propuestas por la American Academy of Dermatology: o, ausente, 1 a 15, leve, 16 a 30, moderado, 31 a 50, grave, y 51 a 8o, muy grave.

Se tomaron fotografías clínicas de todas las uñas con una cámara Lumix ${ }^{\circledR}$ de 10 megapíxeles, con un fondo de color azul turquesa (FIGURA 1).

La valoración ultrasonográfica se llevó a cabo con un con equipo Toshiba Xario ${ }^{\circledR}$, con un transductor lineal de alta resolución que alcanza una frecuencia máxima de $14 \mathrm{MHz}$.

Un radiólogo con experiencia en ultrasonido hizo las mediciones. Al paciente se le advirtió previamente de no usar esmalte ni otros aditamentos en las uñas, ingresó a la cámara de ultrasonido y, en posición sentado, puso la mano dominante en una cubeta con agua a temperatura corporal. La uña y el transductor permanecían sumergidos en el agua, con orientación paralela y un espacio de 5 a $10 \mathrm{~mm}$ entre el transductor y la uña, optimizando la ubicación del punto focal para obtener una adecuada ventana acústica. El refuerzo de interfase posterior producido por el líquido se incrementó y facilitó la capacidad de visualizar las estructuras examinadas.

En los cortes sagitales, se midieron el grosor y el espesor del lecho ungular, la presencia de focos hiperecogénicos en las placas ungulares, los márgenes de las placas y las ondulaciones. Las estructuras anatómicas de las articulaciones interfalángicas distales de la mano y la presencia o ausencia de cambios óseos (erosiones de superficies articulares, osteofitos, osteólisis o aumento del líquido articular), fueron valoradas en los ejes sagital y transversal.

Con ecotomografía Doppler a color se registró el índice de resistencia en el lecho ungular.

Se identificó la presencia de focos hiperecogénicos que comprometieran la placa ventral o dorsal. Se informó borramiento de las placas ungulares cuando había pérdida en la definición de cualquiera de las placas. Para reportar ondulaciones, era necesario documentar compromiso de ambas placas ungulares. Los cambios de sinovitis se definieron como la presencia de líquido que se observa como 


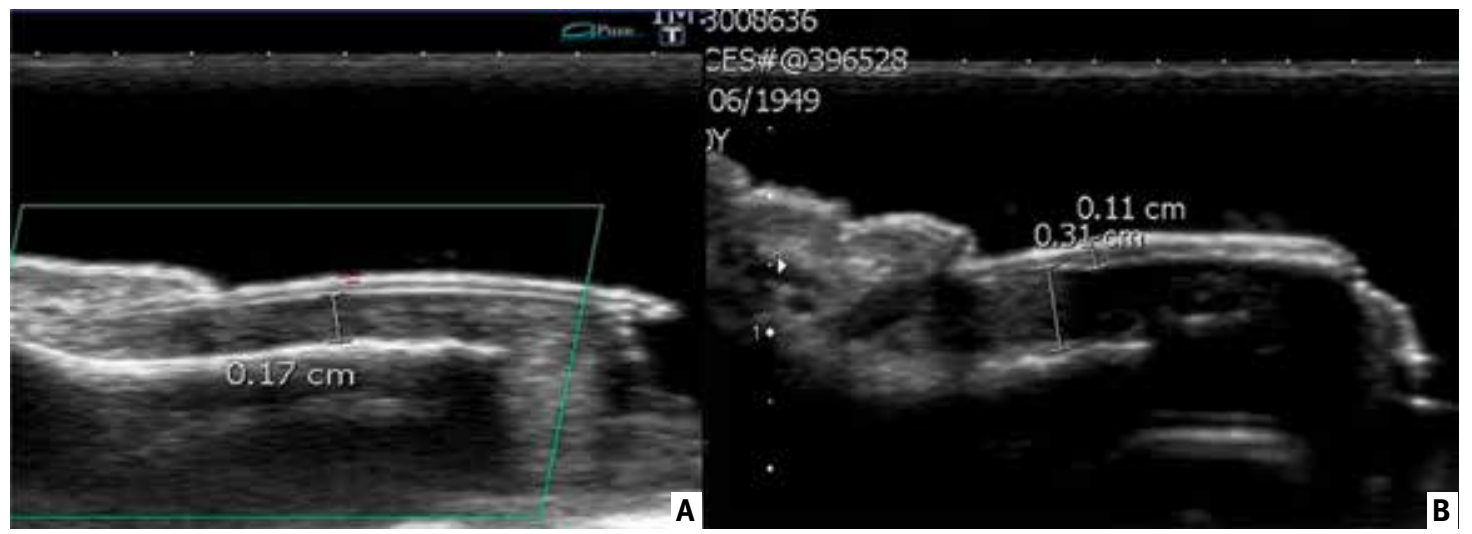

FIGURA 2. A. Lecho y placa ungulares de espesor normal. B. Lecho y placa ungulares engrosados en un paciente con psoriasis ungular.

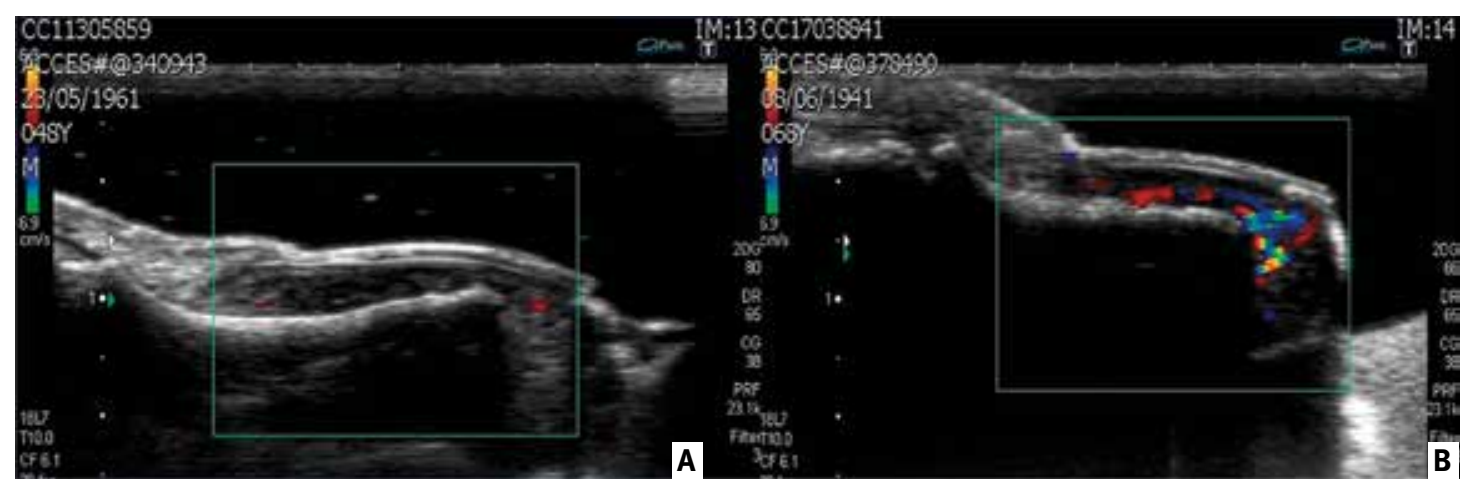

FIGURA 3. Triplex ungular. A. Alta resistencia al flujo arterial en una uña normal. B. Baja resistencia arterial en caso de inflamacion por psoriasis ungular.

fluido anecogénico intraarticular, produciendo ligero abombamiento de la bursa.

La medición del espesor del lecho ungular se obtuvo de la distancia entre la placa ventral ungular y el margen de la cortical ósea de la falange distal, tomando como referencia el tercio medio de la falange distal en el lugar de máxima amplitud, entre la placa ventral y su superficie ósea (FIGURA 2), mientras que el grosor de la placa ungular correspondió a la distancia entre los bordes externos de la placa ungular ventral y la dorsal.

El índice de resistencia arterial, o índice de Pourcelot, se obtuvo midiendo la velocidad pico sistólica menos la velocidad diastólica, dividida por la velocidad sistólica en las curvas espectrales de flujo (FIGURAS 3 Y 4).

Todas las imágenes fueron grabadas en el equipo de ultrasonido y enviadas al sistema de archivo de imágenes IMPAX $^{\circledR} \mathrm{y}$, de este, fueron enviadas en medio magnético (disco compacto); de esta manera, podían ser revisadas posteriormente. El examen duró entre 30 y 40 minutos para la evaluación de la mano dominante.

Se efectuó el estudio anatomo-patológico del grupo de pacientes con psoriasis ungular mediante un corte transversal de la porción distal de la lámina, en el dedo con mayor compromiso, para ser evaluado por un dermatopatólogo. La presencia de paraqueratosis y la tinción negativa con PAS (ácido periódico de Schiff), se consideraron indicativas de psoriasis.

En el laboratorio clínico de la Fundación Santa Fe de Bogotá (certificado por HON@code), se tomaron y analizaron las muestras para HLA B27 a los 31 pacientes que participaron en el estudio.

Empleando el programa estadístico Stata SE, versión 10.1, se hizo un análisis descriptivo de los datos mediante frecuencias absolutas y porcentajes para las variables categóricas y medidas de tendencia central, y de dispersión para las variables cuantitativas. Mediante la prueba de Kruskal-Wallis se compararon en los tres grupos las variables: espesor del lecho ungular, grosor de la placa e índice de resistencia arterial. Se consideró un valor $\mathrm{p}<0,005$ para reportar diferencias estadísticamente significativas.

El protocolo del presente estudio fue aprobado por el Comité de Ética en investigación con seres humanos 


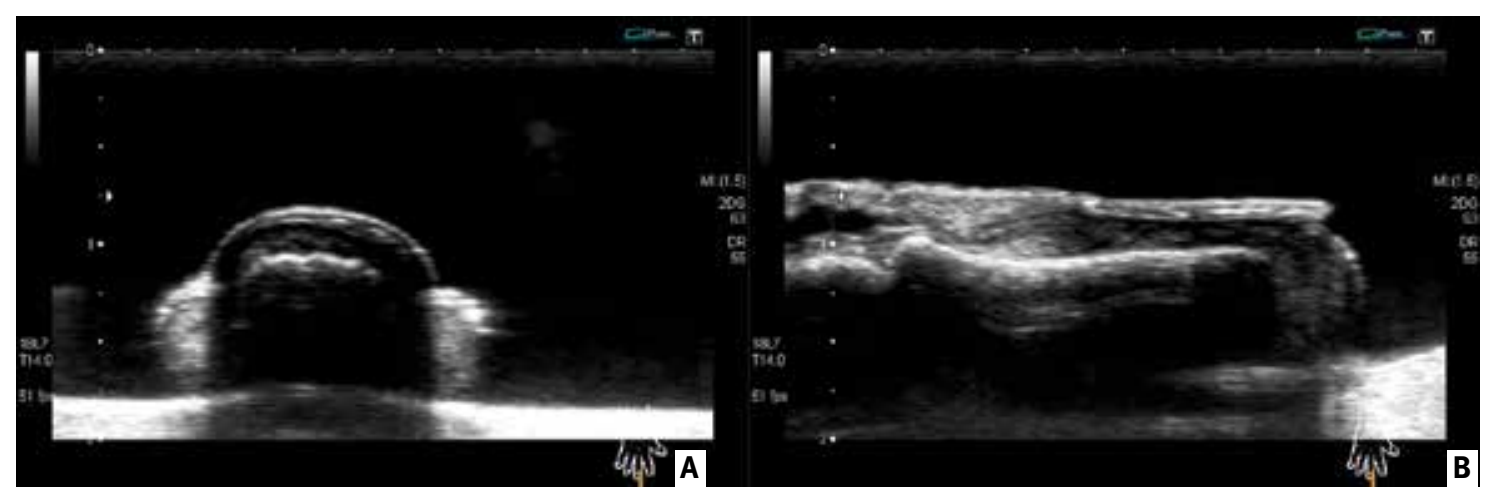

FiguRA 4. Doppler a color. A. Placa y lecho ungulares normales. B. Pérdida de los márgenes de la placa e hiperemia del lecho ungular.

del Hospital de San José y la Fundación Universitaria de Ciencias de la Salud. Los pacientes aceptaron la invitación a participar en el estudio mediante la firma del consentimiento informado.

\section{RESULTADOS}

La mediana de la edad en pacientes con psoriasis ungular fue de 55 (RIQ de 51 a 64) años, en los casos de psoriasis cutánea, fue de 48 (RIQ de 41 a 54) y, en los controles sanos, de 30 (RIQ de 27 a 38). En los pacientes con psoriasis ungular, el tiempo mediano de evolución de la enfermedad fue mayor que en aquellos con psoriasis cutánea: 15 (RIQ de 8 a 26) años versus 4 (RIQ de 2 a 8) años. El índice de gravedad NAPSI en el grupo de pacientes con psoriasis ungular, fue leve en $6 \mathrm{y}$ moderado en 5 (TABLA 1).

Para el reporte de los resultados de la ultrasonografía se consideró como unidad de análisis cada una de las 155 uñas examinadas. Se hallaron cambios óseos en $67,3 \%$ de las unidades analizadas dentro del grupo con psoriasis ungular, en contraste con el $28 \%$ y $26 \%$ de unidades con cambios óseos encontradas en el grupo con psoriasis cutánea y en el de sujetos sanos, respectivamente. En cuanto a la sinovitis, la proporción de unidades afectadas dentro de los grupos con psoriasis ungular y con forma cutánea fue 36,4 \% y $34 \%$, respectivamente; en los sujetos sanos, el porcentaje de cambios de sinovitis fue menor, $16 \%$. Entre los pacientes con psoriasis ungular se encontró hiperecogenicidad en 65,5\% de las unidades analizadas; en el grupo con psoriasis sin compromiso ungular y en los pacientes sanos, el porcentaje fue similar (20\%). La proporción de unidades con márgenes ausentes se distribuyó entre los grupos de la siguiente forma: psoriasis ungular, 38,2 \%, psoriasis cutánea, $16 \%$, y sujetos sanos, $8 \%$. El porcentaje de uñas con ondulaciones fue de $45,5 \%$ en los casos de psoriasis ungular, de $20 \%$ en aquellos con psoriasis cutánea y de $14 \%$ en los sujetos sanos (TABLA 2).

La mediana del espesor del lecho ungular fue mayor entre los grupos con psoriasis (ungular, de 1,9 mm, con RIQ de 1,6 a 2,2; y cutánea, de 1,9 mm, con RIQ de 1,7 a 2,1), comparada con la de los sujetos sanos (1,7 mm, con RIQ de 1,5 a 1,9]) (p<0,01). El grosor de la placa ungular tuvo diferencias significativas entre los grupos $(\mathrm{p}<0,01)$ : en psoriasis ungular, de $0,7 \mathrm{~mm}$ con RIQ de 0,7 a 0,9; en psoriasis cutánea, de o,8 mm con RIQ de 0,7 a 0,9; y en sujetos sanos, de o,6 mm con RIQ de 0,6 a o,7. En el índice de resistencia arterial, no hubo diferencias estadísticamente significativas ( $\mathrm{p}=0,12)$ (TABLA 2).

En el estudio anatomopatológico de la placa ungular de los 11 pacientes con psoriasis ungular, se observó paraqueratosis, y la tinción con PAS fue negativa. El HLA B27 fue positivo en un paciente del grupo sin psoriasis.

\section{DISCUSIÓN}

La evaluación ultrasonográfica se enfocó en las variables de grosor de la placa ungular, el espesor del lecho, las márgenes, las ondulaciones ungulares y la resistencia al flujo ungular.

A pesar de que la mediana del tiempo de evolución de la enfermedad en el grupo con psoriasis ungular, fue de 15 años, el índice NAPSI se reportó entre leve y moderado. Se encontraron diferencias estadísticamente significativas en el grosor y el espesor de la placa ungular. Wortsman, et al., también describieron estos resultados ${ }^{10,11}$, que se podrían interpretar como hallazgos tempranos en la fisiopatología de la psoriasis.

Los cambios óseos fueron más frecuentes en el grupo con psoriasis ungular, hecho que permite confirmar la hipótesis propuesta por otros autores acerca de que este 


\begin{tabular}{|c|c|c|c|}
\hline & $\begin{array}{c}\text { PSORIASIS } \\
\text { UNGULAR * } \\
n=11\end{array}$ & $\begin{array}{c}\text { PSORIASIS } \\
\text { CUTÁNEA † } \\
n=10\end{array}$ & $\begin{array}{c}\text { SANOS } ¥ \\
n=10\end{array}$ \\
\hline Sexo masculino, $\mathrm{n}$ & $7 / 11$ & $4 / 10$ & $4 / 10$ \\
\hline Edad, años mediana (RIQ) § & $55(51-64)$ & $48(41-54)$ & $30(27-38)$ \\
\hline Tiempo de evolución, años mediana RIQ & $15(8-26)$ & $4(2-8)$ & NA \\
\hline Mínimo-máximo & $2,5-55$ & $2-4,5$ & NA \\
\hline \multicolumn{4}{|l|}{ NAPSI } \\
\hline Ausente & & $10 / 10$ & $10 / 10$ \\
\hline Leve & $6 / 11$ & & \\
\hline Moderado & $5 / 11$ & & \\
\hline \multicolumn{4}{|l|}{ Grave } \\
\hline \multicolumn{4}{|l|}{ TRATAMIENTO } \\
\hline Farmacológico & $11 / 11$ & $6 / 10$ & NA \\
\hline Oral & $4 / 11$ & $3 / 10$ & NA \\
\hline Tópico & $6 / 11$ & $6 / 10$ & NA \\
\hline Corticoides & $4 / 11$ & $6 / 10$ & NA \\
\hline Retinoides & $1 / 11$ & $1 / 10$ & NA \\
\hline Fototerapia & $4 / 11$ & $3 / 10$ & NA \\
\hline HLA B27 & 0 & 0 & 1 \\
\hline
\end{tabular}

^ Pacientes con psoriasis ungular. † Pacientes con psoriasis cutánea. ‡ Sujetos sanos. § RIQ: rango intercuartílico. NAPSI: o, ausente; 1 a 15, leve; 16 a 30, moderado; 31 a 50, grave; 51 a 80, muy grave. ๆI NA: no aplica.

TABLA 1. Caracterización de la población.

proceso inflamatorio crónico se relaciona con la matriz ungular y la articulación interfalángica distal, incluyendo su tendón extensor, que se inserta con varios de sus haces en el tejido óseo ${ }^{5,12}$.

Los hallazgos de sinovitis documentados por la ultrasonografía, fueron más frecuentes en los grupos con psoriasis, lo cual sugiere un compromiso músculo-esquelético subclínico, datos que concuerdan con lo reportado por el grupo de Naredo, et $a l^{13}$. No se puede excluir que estos hallazgos se presenten en pacientes sometidos a traumas o que sufran otras enfermedades.

Los cambios en los márgenes y las ondulaciones de las placas ungulares encontrados, pueden corresponder a una alteración en la matriz por procesos inflamatorios adyacentes en el tendón extensor, que se encuentra íntimamente relacionado con las células de la matriz que dan origen a la placa ungular ventral y a la dorsal ${ }^{4,10}$.

Con respecto al índice de resistencia arterial, no se encontraron diferencias significativas entre los tres grupos. En la psoriasis el componente microvascular parece jugar un papel importante en su fisiopatogenia ${ }^{14}$.

El estudio anatomo-patológico realizado a los pacientes con psoriasis ungular, apoyó el diagnóstico clínico en todos los casos.

Con los resultados del presente estudio, se puede observar que la ultrasonografía de alta resolución permite visualizar las anomalías estructurales del aparato ungular y osteoarticular, aportando información de cambios incipientes en la articulación interfalángica distal. Utilizada como herramienta diagnóstica, apoya y complementa la evaluación clínica de los pacientes con psoriasis, con grandes expectativas de utilidad en la fase diagnóstica temprana, y en el seguimiento y tratamiento de la enfermedad.

Aunque el presente estudio no permite predecir el desarrollo de la artritis psoriásica a partir de los cambios ultrasonográficos percibidos, se puede establecer que hay asociación de los hallazgos clínicos en la matriz y el lecho ungular con los hallazgos ultrasonográficos, tal como lo han hecho otros estudios ${ }^{15}$. 


\begin{tabular}{|c|c|c|c|c|c|c|}
\hline & \multicolumn{2}{|c|}{$\begin{array}{l}\text { PSORIASIS UNGULAR } \\
\qquad \mathrm{N}=55\end{array}$} & \multicolumn{2}{|c|}{$\begin{array}{c}\text { PSORIASIS SIN } \\
\text { COMPROMISO UNGULAR } \\
N=50\end{array}$} & \multicolumn{2}{|c|}{$\begin{array}{l}\text { SANOS } \\
\mathrm{N}=50\end{array}$} \\
\hline Cambios óseos, n (\%) & \multicolumn{2}{|c|}{$37(67,3)$} & \multicolumn{2}{|c|}{$14(28,0)$} & \multicolumn{2}{|c|}{$13(26,0)$} \\
\hline Cambios de sinovitis, $\mathrm{n}(\%)$ & \multicolumn{2}{|c|}{$20(36,4)$} & \multicolumn{2}{|c|}{$17(34,0)$} & \multicolumn{2}{|c|}{$8(16,0)$} \\
\hline \multicolumn{7}{|l|}{ HIPERECOGENICIDAD UNGULAR, N (\%) } \\
\hline Presente & 36 & $(65,5)$ & 10 & $(20,0)$ & 10 & $(20,0)$ \\
\hline Márgenes ungulares ausentes, n (\%) & 21 & $(38,2)$ & 8 & $(16,0)$ & 4 & $(8,0)$ \\
\hline Presencia de ondulaciones ungulares, $\mathrm{n}(\%)$ & 25 & $(45,5)$ & 10 & $(20,0)$ & 7 & $(14,0)$ \\
\hline \multicolumn{7}{|l|}{ GROSOR DE LA PLACA, MM } \\
\hline Mediana (RIQ t) & 0,7 & $(0,7-0,9)$ & 0,8 & $(0,7-0,9)$ & 0,6 & $(0,6-0,7)$ \\
\hline Mínimo, máximo & 0,4 & 1,4 & 0,4 & 1,2 & 0,4 & 0,9 \\
\hline \multicolumn{7}{|l|}{ Espesor del lecho ungular, $\mathrm{mm}$} \\
\hline Mediana (RIQ †) & 1,9 & $(1,6-2,2)$ & 1,9 & $\left(1,7^{-}, 2,1\right)$ & 1,7 & $(1,5-1,9)$ \\
\hline Mínimo, máximo & 1,2 & 3,3 & 1,1 & 2,9 & 1,0 & 2,3 \\
\hline \multicolumn{7}{|l|}{ ÍNDICE DE RESISTENCIA ARTERIAL } \\
\hline Mediana (RIQ $t$ ) & 0,53 & $(0,49-0,61)$ & 0,54 & $(0,5-0,59)$ & 0,535 & $(0,49-0,58)$ \\
\hline mínimo, máximo & 0,3 & 0,86 & 0,39 & 0,82 & 0,41 & 0,7 \\
\hline
\end{tabular}

† RIQ: rango, unidad de análisis: uñas.

Datos disponibles sobre 50 uñas de sujetos sanos, 55 uñas de pacientes con psoriasis ungular y 50 uñas de pacientes con psoriasis sin compromiso ungular.

TABLA 2. Análisis ultrasonográfico de uñas de la mano dominante.

Para el análisis de los resultados se debe tener en cuenta que los pacientes con psoriasis ungular se encontraban en tratamiento farmacológico antes del inicio del estudio, por lo que no se puede determinar la influencia del tiempo y la eficacia del tratamiento en los hallazgos de la ultrasonografía, dado que los pacientes se evaluaron de forma transversal. Entre las limitaciones del estudio hay que considerar que se incluyeron 155 unidades de análisis, correspondientes a 31 pacientes. El estudio fue praccticado con un equipo de alta resolución que alcanza una frecuencia máxima de $14 \mathrm{MHz}$.

En conclusión, en pacientes con psoriasis, la ultrasonografía del aparato ungular aporta información sobre cambios que no son aparentes en el examen físico. Estos hallazgos podrían contribuir a la toma de decisiones tempranas. La utilidad de la ultrasonografía ungular está en su uso escalonado, después de aplicar el criterio clínico, que sigue siendo una herramienta útil para el diagnóstico de psoriasis ungular.

\section{AgRADECIMIENTOS}

A Diana Isabel Barrera, médica residente tercer año dermatología, y a la Fundación Universitaria de Ciencias de la Salud, Bogotá, D.C., Colombia. 


\section{REFERENCIAS}

1. Cohen AD, Gilutz H, Henkin Y, Zahger D, Shapiro J, Bonneh DY, et al. Psoriasis and the metabolic syndrome. Acta Derm Venereol 2007;87(6):506-9.

2. González C, Londoño A, Castro L. Guías basadas en la evidencia para el manejo de la psoriasis en Colombia. Editores César González, Angela Londoño, Luis Castro, ASOCOLDERMA, COLPSOR. Impresión Panamericana Formas e Impresos S.A. Bogotá. 2012. p. 23-4.

3. Rich P, Scher RK. Nail Psoriasis Severity Index: A useful tool for evaluation of nail psoriasis. J Am Acad Dermatol. 2003;49:206-12.

4. Jiaravuthisan MM, Sasseville D, Vender RB, Murphy F, Muhn CY. Psoriasis of the nail: Anatomy, pathology, clinical presentation, and a review of the literature on therapy. J Am Acad Dermatol. 2007;57:1-27.

5. McGonagle D, Tan AL, Benjamin M. The nail as a musculoskeletal appendage--implications for an improved understanding of the link between psoriasis and arthritis. Dermatology. 2009;218:97-102.

6. Ruderman EM, Tambar S. Psoriatic arthritis: Prevalence, diagnosis, and review of therapy for the dermatologist. Dermatol Clin. 2004;22:477-86.

7. Gottlieb AB, Chao C, Dann F. Psoriasis comorbidities. J Dermatolog Treat. 2008;19:5-21.

8. Lowes MA, Bowcock AM, Krueger JG. Pathogenesis and therapy of psoriasis. Nature. 2007;445:866-73.
9. Sherlock JP, Joyce-Shaikh B, Turner SP, Chao CC, Sathe M, Grein $\mathrm{J}$, et al. IL-23 induces spondyloarthropathy by acting on RORgammat+ $\mathrm{CD}_{3}+\mathrm{CD}_{4}$-CD8- entheseal resident $\mathrm{T}$ cells. Nat Med. 2012;18:1069-76.

10. Wortsman XC, Holm EZ, Jemec GBE, Gniadecka M, Wulf HC. Ultrasonido de alta resolucion (15 Mhz) en el estudio de la uña psoriática. Santiago. Rev Chil Radiol. 2004;10:6-11.

11. Wortsman X, Jemec GB. Ultrasound imaging of nails. Dermatol Clin. 2006;24:323-8.

12. Gisondi P, Idolazzi L, Girolomoni G. Ultrasonography reveals nail thickening in patients with chronic plaque psoriasis. Arch Dermatol Res. 2012;304:727-32.

13. Naredo E, Moller I, De Miguel E, Batlle-Gualda E, Acebes C, Brito E, et al. High prevalence of ultrasonographic synovitis and enthesopathy in patients with psoriasis without psoriatic arthritis: A prospective case-control study. Rheumatology (Oxford). 2011;50:1838-48.

14. Restrepo JP, Gutiérrez M, De Angelis R, Bertolazzi C, Grassi W. Utilidad de la videocapilaroscopia de lecho ungular en el diagnóstico de enfermedades reumáticas Utility of nailfold videocapillaroscopy in the diagnosis of rheumatic diseases. Rev Asoc Colomb Dermatol. 2009;17:154-61.

15. Ash ZR, Tinazzi I, Gallego CC, Kwok C, Wilson C, Goodfield M, et al. Psoriasis patients with nail disease have a greater magnitude of underlying systemic subclinical enthesopathy than those with normal nails. Ann Rheum Dis. 2012;71:553-6. 